\title{
LETTER
}

\section{Impact of structured care systems on mortality in intensive care units}

\author{
Job van Steenkiste ${ }^{1 *} \mathbb{D}$, Sarah Larson ${ }^{2}$, Erwin Ista ${ }^{3,4}$, Mathieu van der Jagt ${ }^{1}$ and Robert D. Stevens ${ }^{5}$
}

๑ 2021 Springer-Verlag GmbH Germany, part of Springer Nature

\section{Dear Editor,}

The intensive care unit (ICU) is a data-rich environment requiring complex decisions made in the setting of high uncertainty. One approach to reduce complexity and support decision-making is to rationalize care delivery via Structured Care Systems (SCS), defined here as evidence-based guidelines, quality improvement programs, bundles, protocols, or decision algorithms consisting of at least two linked decision-points and/or interventions [2]. It is assumed that SCS have a favorable impact on clinical outcomes and care delivery by reducing variability of clinical practices, increasing efficiency and safety, and better aligning evidence and practice $[1,3,5]$. However, the impact of ICU-based SCS on clinical outcomes is not well established [4]. We aimed to determine the effect of SCS on mortality and length of stay (LOS) in the ICU, accounting for SCS type and target.

We conducted a systematic review and meta-analysis (PROSPERO: CRD42020193517) of prospective studies in adult ICU patients that implemented a SCS, had a control group without the SCS, and reported mortality. The primary outcome was hospital mortality (or another short-term mortality measure if unavailable). Secondary outcomes were LOS in ICU, SCS adherence, and number of Effective Practice and Organisation of Care (EPOC) implementation strategies used. Meta-analysis was completed with raw data and using a random-effects model. Study quality was assessed with the Cochrane Collaboration's risk of bias tool for randomized studies and the Newcastle-Ottawa Scale (NOS) for non-randomized studies. Sensitivity analysis was conducted to identify differences in outcome associated with SCS type and target, and to assess the impact of implementation strategies, study quality, and reported post-implementation adherence. Additional information is in the online supplement.

Results are summarized in Table 1 and in the supplement. We identified 64 studies (1,358,054 patients) published between 1998 and 2020. SCS implementation was associated with a significant reduction in mortality [pooled mortality risk ratio (RR) 0.88 (95\% CI 0.84-0.92, $\left.p<0.001 ; I^{2}=80.85 \%\right)$ ], although effect size was moderate (number needed to treat $=18$ ) and was associated with lower study quality (Table 1). Sensitivity analysis revealed that SCS targeting sepsis or sedation had the largest impact on mortality [respectively, RR 0.73 (95\% CI $0.65-0.81, p<0.001)$ and RR 0.86 (95\% CI 0.76-0.99, $p=0.02)$ ]. Among the individual SCS types, care bundles (RR 0.82, 95\% CI 0.76-0.89, $p \leq 0.001$ ) and guidelines (RR $0.86,95 \%$ CI $0.77-0.97, p=0.01$ ) were significantly associated with mortality reduction. No relation was noted between SCS and ICU-LOS. Level of SCS adherence was reported in 29 studies (45\%) and the median post-implementation adherence rate was 83\% (IQR $42-91 \%$ ). Sensitivity analysis found that neither adherence rate nor the number of EPOC implementation strategies was significantly associated with mortality.

*Correspondence: jobvansteenkiste@gmail.com

${ }^{1}$ Department of Intensive Care Adults, Erasmus MC, University Medical Center, P.O. Box 2040, Rotterdam CA 3000, The Netherlands Full author information is available at the end of the article Job van Steenkiste and Sarah Larson contributed equally to this work.

\section{至 Springer}


Table 1 Meta-analysis and sensitivity analysis of the primary mortality outcome as effect measure for SCS type and target, adherence, implementation strategies, and quality assessment

\begin{tabular}{|c|c|c|c|c|}
\hline Comparison & Number of studies & Risk ratio & 95\% confidence interval & $p$ value \\
\hline Overall meta-analysis for mortality & 64 & 0.88 & $0.84-0.92$ & $<0.001$ \\
\hline \multicolumn{5}{|l|}{ SCS type $(n=64)$} \\
\hline Bundles & 24 & 0.82 & $0.76-0.89$ & $<0.001$ \\
\hline Protocols & 19 & 0.94 & $0.81-1.09$ & 0.400 \\
\hline Guidelines & 9 & 0.86 & $0.77-0.97$ & 0.011 \\
\hline Quality improvement programs & 6 & 1.01 & $0.92-1.10$ & 0.865 \\
\hline Algorithms & 4 & 0.86 & $0.64-1.16$ & 0.317 \\
\hline \multicolumn{5}{|l|}{ SCS target $(n=37)$} \\
\hline Sepsis & 11 & 0.73 & $0.65-0.81$ & $<0.001$ \\
\hline Ventilator-associated pneumonia & 7 & 0.95 & $0.83-1.09$ & 0.441 \\
\hline Weaning from mechanical ventilation & 7 & 1.27 & $0.95-1.69$ & 0.103 \\
\hline Sedation & 4 & 0.86 & $0.76-0.98$ & 0.022 \\
\hline Pain Agitation Delirium & 4 & 0.76 & $0.54-1.07$ & 0.116 \\
\hline Nutrition & 4 & 0.89 & $0.72-1.11$ & 0.316 \\
\hline \multicolumn{5}{|l|}{ Adherence $(n=29)$} \\
\hline$\geq 83.3 \%$ adherence $^{\mathrm{a}}$ & 15 & 0.90 & $0.80-1.02$ & 0.096 \\
\hline$<83.3 \%$ adherence ${ }^{a}$ & 14 & 0.81 & $0.74-0.88$ & $<0.001$ \\
\hline \multicolumn{5}{|l|}{ Implementation strategies $(n=64)$} \\
\hline$\geq 6$ EPOC strategies used ${ }^{\mathrm{a}}$ & 37 & 0.89 & $0.84-0.94$ & $<0.001$ \\
\hline$<6$ EPOC strategies used & 27 & 0.89 & $0.80-0.98$ & 0.021 \\
\hline \multicolumn{5}{|l|}{ Quality assessment $(n=64)$} \\
\hline Randomized studies overall & 14 & 0.95 & $0.81-1.11$ & 0.514 \\
\hline Randomized studies 'low risk of bias' & 9 & 1.08 & $0.90-1.31$ & 0.416 \\
\hline Randomized studies 'some concerns of bias' & 5 & 0.72 & $0.59-0.88$ & $<0.001$ \\
\hline Non-Randomized studies overall & 50 & 0.87 & $0.83-0.92$ & $<0.001$ \\
\hline Non-Randomized studies $\geq 7$ NOS stars & 35 & 0.90 & $0.85-0.95$ & $<0.001$ \\
\hline Non-Randomized studies $<7$ NOS stars & 15 & 0.78 & $0.68-0.89$ & $<0.001$ \\
\hline
\end{tabular}

NOS New Ottawa Scale, EPOC Effective Practice and Organization of Care

a Interpretation: studies comparing populations with and without exposure to the structured care systems (SCS) with either higher or lower than the median reported post-implementation adherence rate of $83.3 \%$ among 29 studies that reported adherence data. Similarly for EPOC strategies, maximum number of NOS stars is nine; seven stars indicates good or fair quality

Results support deployment of SCS in the ICU, specifically bundles and guidelines or those targeting sepsis or sedation. No association was found between mortality and SCS adherence or use of specific implementation strategies, suggesting that additional research is needed on potential modifiers of the relationship between SCS and mortality. Studies are also needed to identify components, implementation strategies, and target populations which maximize the impact of SCS on outcomes of critically ill patients.
Supplementary Information

The online version contains supplementary material available at https://doi. org/10.1007/s00134-021-06383-5.

\section{Author details}

${ }^{1}$ Department of Intensive Care Adults, Erasmus MC, University Medical Center, P.O. Box 2040, Rotterdam CA 3000, The Netherlands. ${ }^{2}$ Department of International Development, The London School of Economics and Political Science, Houghton St, Holborn, London WC2A 2AE, UK. ${ }^{3}$ Nursing Science, Department of Internal Medicine, Erasmus MC, University Medical Center, P.O. Box 2040, Rotterdam CA 3000, The Netherlands. ${ }^{4}$ Pediatric Intensive Care, Erasmus MC-Sophia Children's Hospital, University Medical Center, P.O. Box 2040, Rotterdam CA 3000, The Netherlands. ${ }^{5}$ Department of Anesthesiology and Critical 
Care Medicine, Johns Hopkins University School of Medicine, Baltimore, MD, USA.

\section{Acknowledgements}

We thank Wichor Bramer, biomedical Information specialist, for guiding the literature search at the Erasmus University Medical Library. We would also like to thank Gayane Yenokyan from the Department of Biostatistics, Johns Hopkins Bloomberg School of Public Health, for her critical review of the meta-analysis.

\section{Declarations}

\section{Conflicts of interest}

On behalf of all authors, the corresponding author states that there is no conflict of interest.

\section{Publisher's Note}

Springer Nature remains neutral with regard to jurisdictional claims in published maps and institutional affiliations.

Received: 4 February 2021 Accepted: 12 March 2021
Published online: 27 March 2021

\section{References}

1. Borgert MJ, Goossens A, Dongelmans DA (2015) What are effective strategies for the implementation of care bundles on ICUs: a systematic review. Implement Sci 10:119

2. Kavanagh BP, Nurok M (2016) Standardized Intensive Care. Protocol misalignment and impact misattribution. Am J Respir Crit Care Med 193(1):17-22. https://doi.org/10.1164/rccm.201502-0314CP

3. Sevransky JE, Agarwal A, Jabaley CS, Rochwerg B (2021) Standardized care is better than individualized care for the majority of critically III patients. Crit Care Med 49(1):151-155. https://doi.org/10.1097/CCM. 0000000000004676

4. Wall RJ, Dittus RS, Ely EW (2001) Protocol-driven care in the intensive-care unit: a tool for quality. Crit Care 5(6):283-285

5. Woolf SH, Grol R, Hutchinson A, Eccles M, Grimshaw J (1999) Clinical guidelines: potential benefits, limitations, and harms of clinical guidelines. BMJ 318(7182):527-530 